\title{
A Diabetic Healthcare Recommendation System
}

\author{
Sanket Bankhele \\ Dept. of Information \\ Technology \\ PCCOE, Pune, India
}

\author{
Ashish Mhaske \\ Dept. of Information \\ Technology \\ PCCOE, Pune, India
}

\author{
Shikha Bhat \\ Dept. of Information \\ Technology \\ PCCOE, Pune, India
}

\author{
S. V. Shinde, PhD \\ Dept. of Information \\ Technology \\ PCCOE, Pune, India
}

\begin{abstract}
Recently there has been an increase in the number of diabetic patients at an alarming rate. Approximately 18 million people die from cardiovascular diseases every year where diabetes is one of the major factor. Treating diabetes and monitoring it is required to efficiently manage health conditions of diabetic patients. In this paper, an android application has been designed and developed that recommends probable medication, diet and exercise to help people manage their diabetes well. This system analyses the input parameters that are entered by the end user and provides personalized services for users in the form of recommendations for their diet, medicines and exercises. This android-based system can also remind users to carry out the recommendations, which are provided by the system. Other than the functional features, there are also several important non-functional features of the extensibility and the convenience for use. The recommendation is done using User based collaborative filtering, the system asks the user to enter a predetermined set of parameters which are matched with other patients parameters stored in the database, the database consists of past cases of patients who have been diagnosed with diabetes and treated, this matching is done using Pearson Correlation, the matched patient's diet, exercise and workout is then recommended to the current user.
\end{abstract}

\section{General Terms}

Recommender System

\section{Keywords}

User based Collaborative Filtering, Pearson Correlation Score

\section{INTRODUCTION}

People nowadays can easily get their physical examination done in a hospital and get the reports of various attributes like triceps skinfold thickness, Body mass index, glucose and insulin levels which can be used to analyze their diabetic health and also manage it. In this paper, an android healthcare recommendation system has been designed and developed to help people, especially for those who suffer from diabetes to easily understand their health conditions and then well manage it. This system analyses the parameters that the user enters, finds the patient's record from the database with most similar attributes and provides service to the current user in terms of probable medicines, diet and exercise guideline recommendations [3]. The android-based system can also regularly remind users to carry out the recommendations, which are provided by the system.

\section{MOTIVATION}

Diabetes is a lethal disease that needs to be addressed and controlled. It is not as dangerous as other diseases like HIV, malaria, but diabetes is a disease where most people are globally diagnosed with, than all of the above mentioned diseases combined [1]. There is an estimation that says 415 million people are diagnosed with diabetes worldwide which has added 33 million people more than in 2013.There has been an exponential increase in the use of internet technology, and people have become almost completely dependent on it. Today, people prefer various websites that suggest diets, workouts, to manage their health. They can get lot of information from such sites, however the available information may not be personalized with respect to every individual. Also various research domains are working in the field of medical research [4],[5]. Because everybody has his/her own health status and it is very common that one healthcare guideline recommendation may be very helpful to somebody, but it may be helpless, even noxious to others. A diet plan and workout of people with similar attributes or parameters like age, BMI can be recommended and be useful.

\section{RECOMMENDER SYSTEMS}

\subsection{Recommender Systems}

Recommender systems in general predict user preferences so as to suggest items to purchase. A recommendation system can simply be a system that recommends you things that are similar to your past purchases or may be videos watched by other similar users etc. Recommendation systems focus on predictions based on similarity measures. Many techniques have been used for recommendation tasks, which consist of collaborative recommender systems, content-based recommender systems, knowledge-based recommender systems and various other techniques.

\subsection{Collaborative Filtering systems}

Collaborative filtering is the most acclaimed approach for providing personalized recommendations. Various admired websites are available that make use of the collaborative filtering technology include Amazon, Netflix, iTunes, IMDB, LastFM, Delicious and StumbleUpon. In collaborative filtering, algorithms are used to make automatic predictions about a user's interests by compiling preferences from several users [2]. Following are the types of recommendation systems:

User-based: finding similar users to the current user at their likes and dislikes to predict interests of the current user.

Item-based: use same data but find items near current item.

Content-based: use different data- find items with similar properties.

\subsection{User-based Collaborative filtering}

Currently, the most personalized recommendation technology that is used in filtering algorithms is the user-based collaborative filtering method. In Collaborative Filtering for example, if two users have purchased and rated the same item and if the rating by both the users is same, then the next product that user $\mathrm{U} 1$ buys and rates it, the same rating would be predicted for user $\mathrm{U} 2$ for the same product [2]. This 
similarity needs to be computed for all the user ratings, which can be done using:

\section{User based Collaborative}

-For each user on list, $U 1$

-For each product $P$ rated by $U 1$

-For each user $U 2$ rate product $P$

-Save that a product rated by $U 1$ and $U 2$

-For each user U2

-Compute similarity between $\mathrm{U} 1$ and $\mathrm{U} 2$

\section{USER SIMILARITY}

\subsection{Measuring user similarity between the} users:

A popular similarity measure in user-based collaborative filtering is Pearson Correlation. Correlation between sets of data is a measure of how well they are related. The most common measure of correlation is the Pearson Correlation. The full name is the Pearson Product Moment Correlation or PPMC. It shows the linear relationship between two sets of data. This method returns a value between -1 and 1 , where 1 means the two products have exactly the same ratings.

\subsection{Taking User's parameters for measuring the similarity:}

In order to recommend the users with their diet, medication and exercise a database with the history of the diagnosed and treated patients is maintained which consists of the medication, diet plans and exercise that were used to treat the patient. The parameters including Insulin level, Blood pressure, BMI, age, Glucose level, Triceps Skinfold Thickness are compared with every other user in the database and the patient with the most similar parameters is identified using Pearson Correlation Score.

\subsection{Using Pearson's correlation in matching of the parameters:}

As the user similarity is computed on the basis of similar ratings, in the same way the user parameters are matched with every other user using Pearson Correlation Score and the closest user is identified. The closest user is the one which has the score that is closest to 1 .

-For each user on list, $U 1$

-For each parameter $P$ of User U1

-For each User $U 2$ having parameter $P$

-Save the parameter of $U 1$ and $U 2$

-For each User U2

-Compute Similarity between $U 1$ and $U 2$

\subsection{Recommendations after the}

\section{identification of the closest match:}

Once the parameters are matched and the closest user is found the diet, medication and the exercise of the matched patient is retrieved from the database and is recommended to the user.

\section{PROPOSED SYSTEM}

\subsection{System Overview:}

In the proposed system, the user needs to register to the system where the user is asked to enter a predetermined set of parameters. Once the user is registered to the system, the parameters entered are matched with the existing database so as to calculate the Pearson co-relation score which leads to finding the closest user

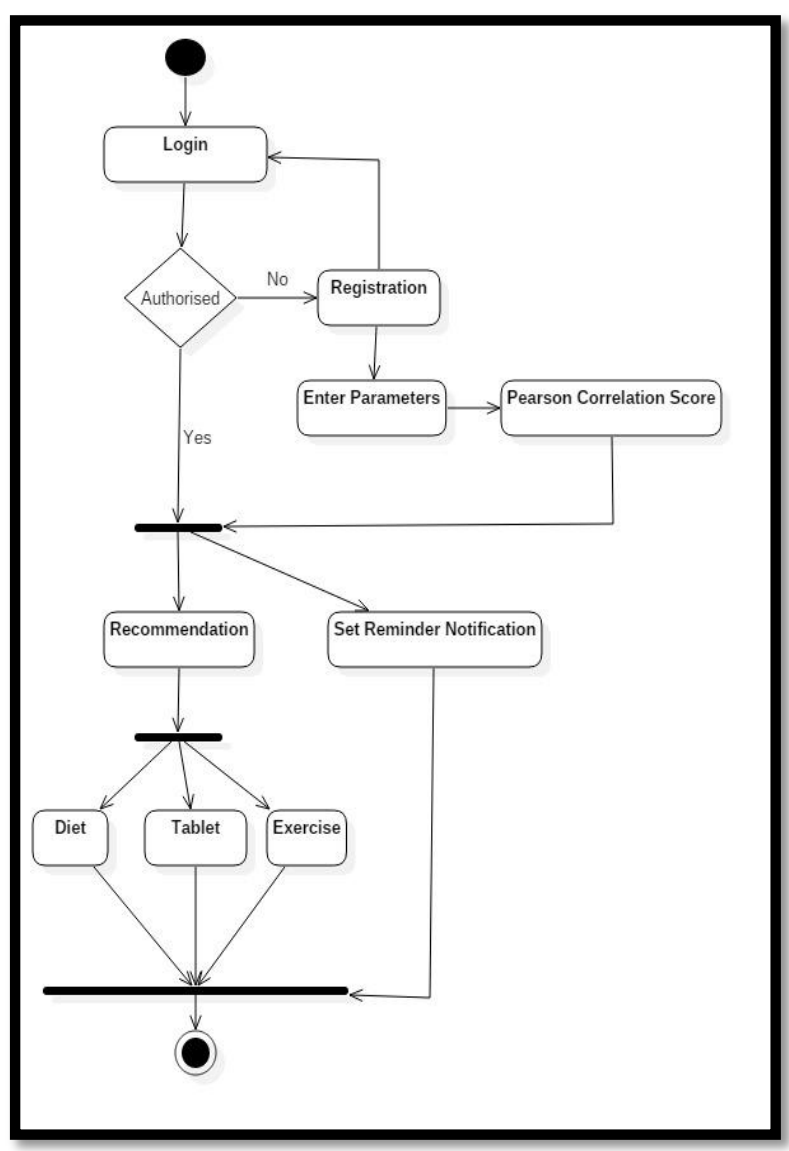

Fig 1: System Architecture

\subsection{Flow of the application:}

Following is the flow of the android application in which user has to register in order to get started.

Fig3. shows the Registration form in which the user is asked to fill certain parameters in order to get registeres to the system. Once the user is registered to the system the user is asked to enter a predetermined set of parameters which are later used to find patients with similar attributes and make the reccommendations [3] .

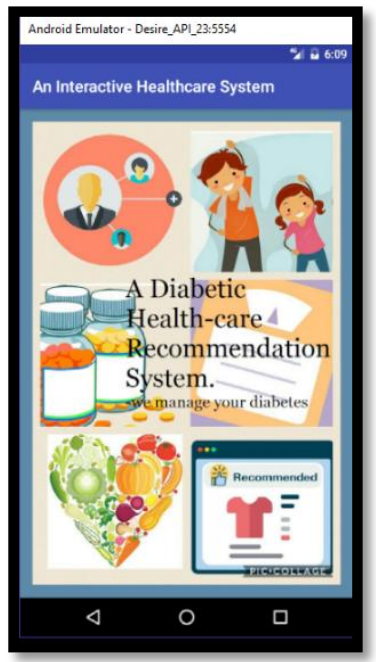

Fig 2: Title Screen 


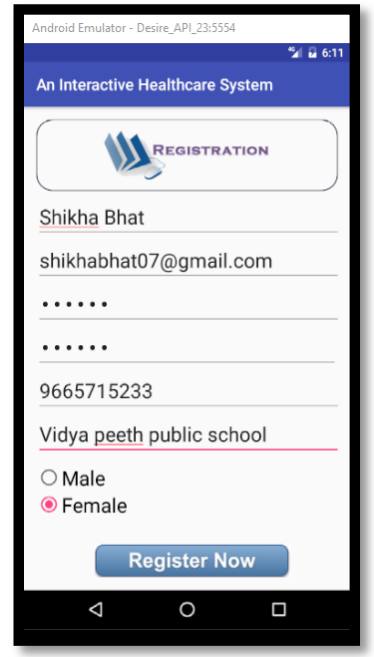

Fig 3: Registration Form

Fig4. Shows the add attribute screen in which the user enters the paramters that are asked and submits the paramters to the system. After the parameters are submitted to the system the system calculates the Pearson score based on which the recommendations are made.

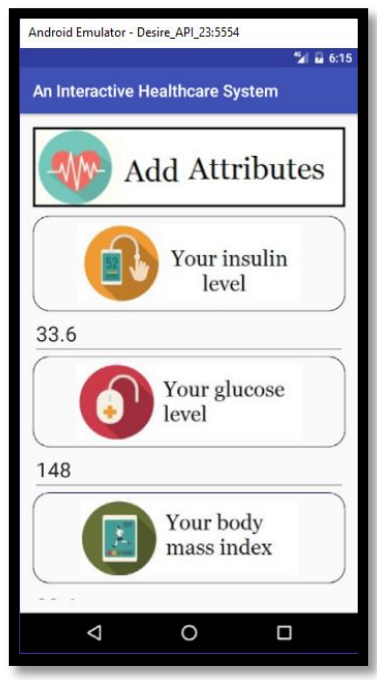

Fig 4: Add Attributes

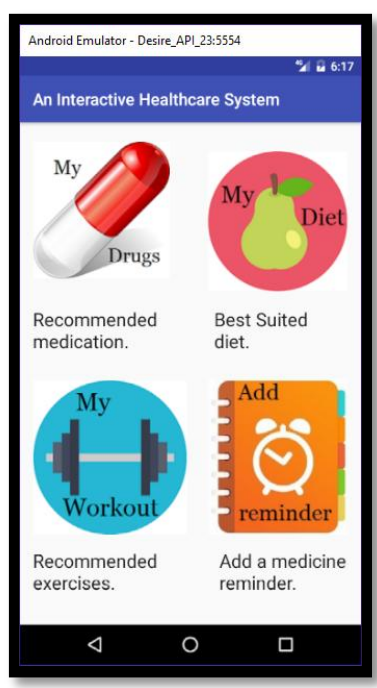

Fig 5: Home Screen
Every time the user logins to the system, the user can acces the home screen as shown in Fig5. which enables the user to view the the recommendations that have been made by the system in the form of exercise guidelines, probable medication and the suited diet. The system also alows the user to set reminders to get notifications and reminders in order to carry out the reccommendations made by the system. On clicking the buttons, the user is navigated to the respective module.

Fig6. shows the medicine reccomendation module which is opened once the user clicks on the button for medicine recommendation on the home screen. The module also reccommends the dosage to be taken.

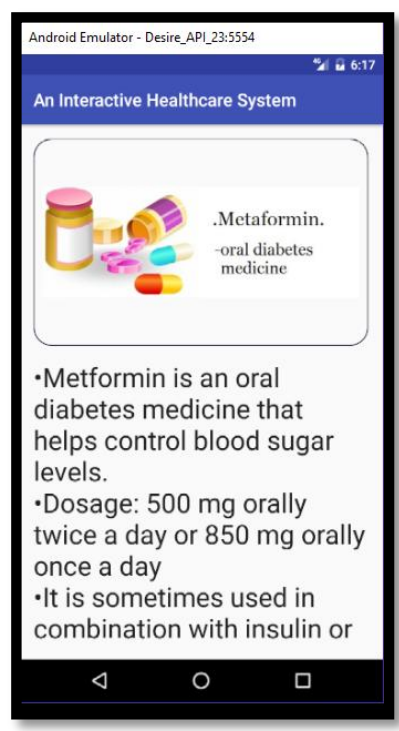

Fig 6: Medicine Recommendation Screen

Fig7. shows the diet reccommendations that are made by the system and the user is navigated to this screen when the user clicks the diet recommendation button on the home screen. This module suggests the user a diet that was used by patients with similar attributes along with quantity and dosage time.

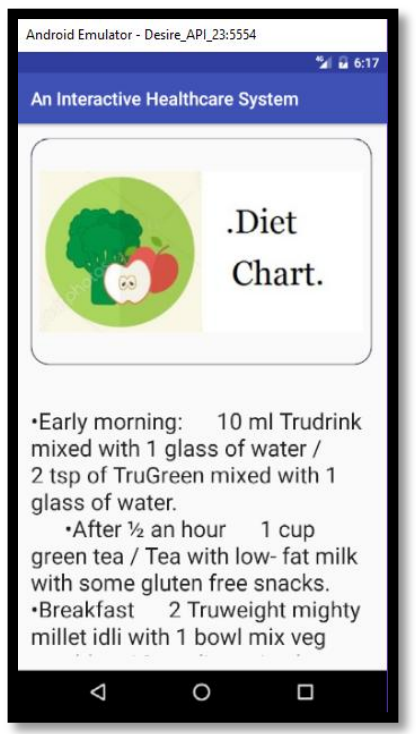

Fig 7: Diet Recommendation Screen

Fig8. shows the exercise reccomendation screen which is displayed when the user clicks on the exercise reccommendation button on the home screen. 


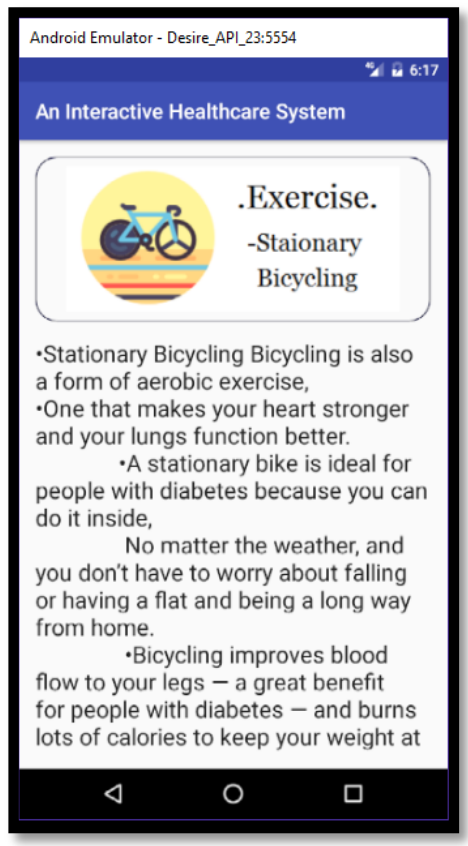

Fig 8: Exercise Recommendation Screen

Fig9. shows the reminder module that allows the user to set and cancel the alarms that can remind the users with a notification of taking the medicines and carrying the recommended exercises.

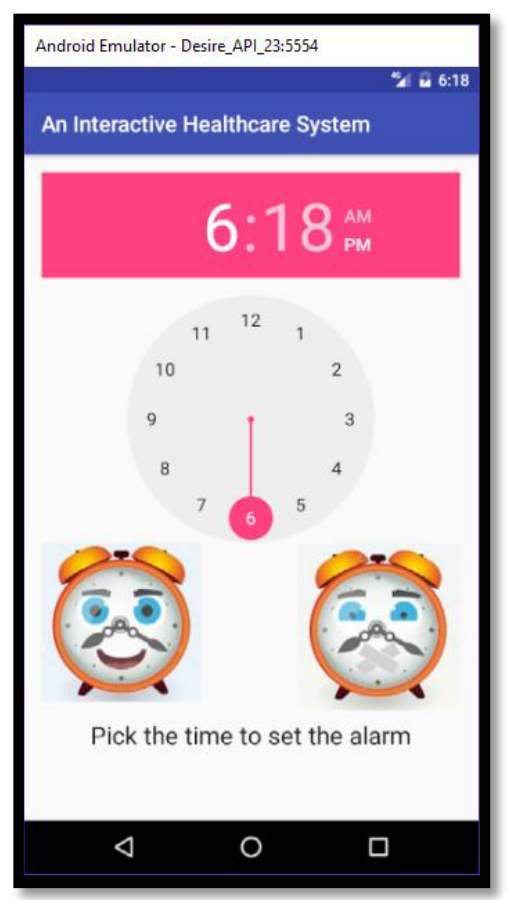

Fig 9: Medicine Reminder Screen

\section{MATHEMATICAL MODEL \\ Pearson Correlation Score:}

Let $\mathrm{a}$ and $\mathrm{b}$ be the users

$\mathrm{r}_{\mathrm{a}, \mathrm{p}}$ : value of user a for attribute $\mathrm{p}$ with $\overline{r_{\mathrm{a}}}$ as mean over set $\mathrm{P}$ of attributes $\mathrm{p}$

$r_{b, p}$ : value of user $b$ for attribute $p$ with $\overline{n_{b}}$ as mean over set $P$ of attributes $p$

$P$ : set of attributes of both the users $a$ and $b$
Formula for finding Pearson Correlation Score:

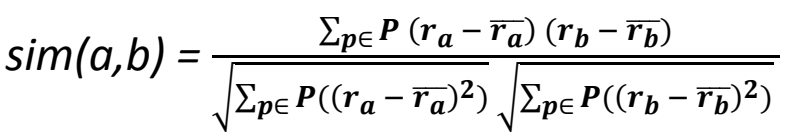

Example- A database of attributes of Alice and some other users is given

Table 1. User Attributes

\begin{tabular}{|l|l|l|l|l|l|l|}
\hline & Age & Insulin & Glucose & BMI & BP & $\begin{array}{l}\text { Triceps } \\
\text { thickness }\end{array}$ \\
\hline Alice & 21 & 23.3 & 89.0 & 28.1 & 66.0 & 23.0 \\
\hline User1 & 31 & 31 & 118 & 45.8 & 84 & 47 \\
\hline User2 & 51 & 25.6 & 166 & 25.8 & 72 & 19 \\
\hline User3 & 26 & 28.1 & 78 & 31 & 50 & 32 \\
\hline User4 & 53 & 43.1 & 197 & 30.5 & 70.0 & 45.0 \\
\hline
\end{tabular}

Table 2. Current User Attributes

\begin{tabular}{|l|l|l|l|l|l|l|}
\hline User & Age & Insulin & Glucose & BMI & BP & $\begin{array}{l}\text { Triceps } \\
\text { thickness }\end{array}$ \\
\hline Alice & 21 & 23.3 & 89.0 & 28.1 & 66.0 & 23.0 \\
\hline
\end{tabular}

In Table1, user attributes are taken into consideration. Table2 shows the current user attributes. In Table3, Pearson Correlation Score is calculated in which all the parameters of other users are matched with the attributes of the current user in Table2.

Table 3. Pearson Score of users with respect to current user attributes.

\begin{tabular}{|l|l|l|l|l|l|l|l|}
\hline User & Age & $\begin{array}{l}\text { Insu } \\
\text { lin }\end{array}$ & $\begin{array}{l}\text { Gluc } \\
\text { ose }\end{array}$ & $\begin{array}{l}\text { BM } \\
\text { I }\end{array}$ & BP & $\begin{array}{l}\text { Trice } \\
\text { ps } \\
\text { Thic } \\
\text { kness }\end{array}$ & $\begin{array}{l}\text { Pearson } \\
\text { Score }\end{array}$ \\
\hline $\begin{array}{l}\text { User } \\
1\end{array}$ & 31 & 31 & 118 & 45.8 & 84 & 47 & 0.84485 \\
\hline $\begin{array}{l}\text { User } \\
2\end{array}$ & 51 & 25.6 & 166 & 25.8 & 72 & 19 & 0.96421 \\
\hline $\begin{array}{l}\text { User } \\
3\end{array}$ & 26 & 28.1 & 78 & 31 & 50 & 32 & 0.34210 \\
\hline $\begin{array}{l}\text { User } \\
4\end{array}$ & 53 & 43.1 & 197 & 30.5 & 70 & 45 & 0.59937 \\
\hline
\end{tabular}

Here the User 2 value is near to current user (Alice), which means that the User 2 and the current user are most similar. So the diet, exercise and medicines of User 2 will be recommended to the current user (Alice).

\section{RESULT ANALYSIS}

This system is a recommendation system in which the user needs to register to the system where the user is asked to enter a predetermined set of parameters. Once the user is registered to the system, the parameters entered are matched with the existing database so as to calculate the Pearson co-relation score which leads to the matching of the parameters and 
finding the closest user. The Pearson correlation similarity score is a metric that measures how highly correlated two variables are and is measured from -1 to +1 . A Pearson Correlation Coefficient of 1 indicates that the data objects are perfectly correlated but in this case, a score of -1 means that the data objects are not correlated. Once the closest user is determined the diet, exercise and medication values that were used for the rehabilitation of that patient are retrieved and recommended to the user. Fig4 is the form which takes the parameters from the user and is given as input to the recommender system and accordingly the similar users are identified and the recommendations are made to the user as shown in Fig6, Fig7, Fig8. The result shows the probable medication, exercise, and diet to the new user. The scope of the project can be extended to other disease diagnosis using the same system and will vary with the database and the parameters of the particular disease that is being diagnosed.

\section{CONCLUSION}

This work presents a Diabetic healthcare system with personalized diet, exercise and medicine recommendations using Collaborative Filtering algorithm. The Collaborative Filtering algorithm can effectively and efficiently recommend the patient along with the most helpful information for individual health management. In this work an interactive system has been developed including the personal health management module and a reminding function for android mobile phones, to let users easily make use of this system. Moreover, the system can be easily extended to other diseases to provide other kinds of healthcare guideline recommendations.

\section{REFERENCES}

[1] Wu, Chun-Hui, Fang, Kwot-Ting, and Chen, Ta-Cheng: Applying data mining for prostate cancer. In: the International Conference on New Trends in Information and Service Sci-ence,pp. 1063-1065, Beijing (2009)

[2] Siavash Ghodsi Moghaddam, Ali Selamat : A scalable Collaborative recommender algorithm based on User Density-based Clustering.

[3] Jerry C.C. Tseng, Bo-Hau Lin, Yu-Feng Lin, An Interactive Healthcare System with Personalized Diet and Exercise Guideline Recommendation.

[4] S. V. Shinde and U. V. Kulkarni, "Mining Classification Rules from Modified Fuzzy Min-Max Neural Network for Data with Mixed Attributes," Elsevier Journal Applied Soft Computing Journal, pp. 364-378, Dec. 2016.

[5] S. V. Shinde and U. V. Kulkarni, Extended Fuzzy Hyperline-Segment Neural Network with Classification Rule Extraction, Article in Press, Neurocomputing, April 2017. 